\title{
A GENERALIZATION OF AN IDENTITY INVOLVING THE INVERSES OF BINOMIAL COEFFICIENTS
}

\author{
ZHIZHENG ZHANG AND HAITAO SONG
}

Abstract. By applying the integral expression of the inverses of binomial coefficients, the authors generalize the Sury's identity.

\section{Introduction}

As usual, the binomial coefficients are defined by

$$
\left(\begin{array}{l}
n \\
m
\end{array}\right)=\left\{\begin{array}{cc}
\frac{n !}{m !(n-m) !} & \text { if } n \geq m, \\
0 & \text { if } n<m,
\end{array}\right.
$$

where $n$ and $m$ are nonnegative integers. Sury [3] used

$$
\left(\begin{array}{l}
n \\
m
\end{array}\right)^{-1}=(n+1) \int_{0}^{1} t^{m}(1-t)^{n-m} d t
$$

to obtain that

$$
\sum_{m=0}^{n} \frac{1}{\left(\begin{array}{c}
n \\
m
\end{array}\right)}=\frac{n+1}{2^{n}} \sum_{m=0}^{n} \frac{2^{m}}{m+1}=\frac{n+1}{2^{n}} \sum_{j \text { odd }} \frac{1}{j}\left(\begin{array}{c}
n+1 \\
j
\end{array}\right)
$$

It's necessary to point out that the first equation of identity (2) is identity (2.25) in Could's collection [1]. Using the integral identity (1), many results have been obtained for the finite and infinite sums related to the reciprocals of binomial coefficients. See [2, 3, 4, 5, 6, 7]. And in [7], Sury, Wang and Zhao generalized identity (2) and obtained a polynomial version.

The purpose of this paper is to extend the identity (2) much further and obtain some interesting results.

Received January 29, 2007; revised May 31, 2007.

2000 Mathematics Subject Classification. 05A10, 05A19.

Key words and phrases. Combinatorial identity, Sury's identity, binomial coefficients. 


\section{Main results}

Theorem 2.1. Let $n, k$, and $r$ be nonnegative integers, then in the ring $Q[T]$ of rational polynomials, the identity

$$
\begin{aligned}
\sum_{m=k}^{n} \frac{T^{m}(1-T)^{n-m}}{\left(\begin{array}{c}
n+r \\
m
\end{array}\right)}= & (n+r+1) \sum_{i=0}^{n-k} \frac{T^{n-i}(1-T)^{n-k+1}}{(r+k+i+1)\left(\begin{array}{c}
r+k+i \\
k
\end{array}\right)} \\
& +(n+r+1) \sum_{i=0}^{r}\left(\begin{array}{c}
r \\
i
\end{array}\right)(-1)^{i} \sum_{p=k}^{n} \frac{T^{n+1}(1-T)^{n-p}}{i+p+1}
\end{aligned}
$$

holds for $k \leq n$. An equivalent form is that for $\lambda \neq-1$,

$$
\begin{aligned}
\sum_{m=k}^{n} \frac{\lambda^{m}}{\left(\begin{array}{c}
n+r \\
m
\end{array}\right)}= & (n+r+1) \sum_{i=0}^{n-k} \frac{\lambda^{k+i}}{(\lambda+1)^{i+1}} \sum_{p=0}^{n+r-k-i}\left(\begin{array}{c}
n+r-k-i \\
p
\end{array}\right)(-1)^{p} \frac{1}{p+k+1} \\
& +(n+r+1) \frac{\lambda^{n+1}}{(\lambda+1)^{n+2}} \sum_{i=0}^{r}\left(\begin{array}{c}
r \\
i
\end{array}\right)(-1)^{i} \sum_{p=k}^{n} \frac{(\lambda+1)^{p+1}}{i+p+1} .
\end{aligned}
$$

Moreover,

$$
\sum_{m=k}^{n} \frac{\lambda^{m}}{\left(\begin{array}{c}
n+r \\
m
\end{array}\right)}=(n+r+1) \sum_{i=0}^{n-k} \lambda^{n-i} \sum_{p=0}^{i+r}\left(\begin{array}{c}
i+r \\
p
\end{array}\right)(-1)^{p} \frac{1}{n+p-i+1}
$$

Proof. For a fixed real number $\lambda$, letting $I_{n, k, r}(\lambda)=\sum_{m=k}^{n} \frac{\lambda^{m}}{\left(\begin{array}{c}n+r \\ m\end{array}\right)}$, it follows from identity (1) that

$$
\begin{aligned}
I_{n, k, r}(\lambda) & =(n+r+1) \int_{0}^{1}(1-t)^{n+r} \sum_{m=k}^{n}\left(\frac{\lambda t}{1-t}\right)^{m} d t \\
& =(n+r+1) \int_{0}^{1}(1-t)^{r}(\lambda t)^{k} \frac{(\lambda t)^{n-k+1}-(1-t)^{n-k+1}}{(\lambda+1) t-1} d t .
\end{aligned}
$$

Putting $s=(\lambda+1) t-1$, we get

$$
I_{n, k, r}(\lambda)=(n+r+1) \frac{\lambda^{k}}{(\lambda+1)^{k+r+1}} \int_{-1}^{\lambda}(\lambda-s)^{r}(s+1)^{k} \frac{\lambda^{n+1-k}(s+1)^{n+1-k}-(\lambda-s)^{n+1-k}}{s(\lambda+1)^{n+1-k}} d s . \text { (6) }
$$

Then we have that $I_{n, k, r}=(n+r+1)\left(I_{1}+I_{2}\right)$, where

$$
I_{1}=\frac{\lambda^{k}}{(\lambda+1)^{n+r+2}} \int_{-1}^{\lambda}(\lambda-s)^{r} \frac{(s+1)^{k}}{s}\left(\lambda^{n+1-k}-(\lambda-s)^{n+1-k}\right) d s
$$

and

$$
I_{2}=\frac{\lambda^{n+1}}{(\lambda+1)^{n+r+2}} \int_{-1}^{\lambda}(\lambda-s)^{r} \frac{(\lambda+1)^{n+1}-(s+1)^{k}}{s} d s
$$

By writting

$$
\frac{(\lambda+1)^{n+1}-(s+1)^{k}}{s}=\sum_{p=k}^{n}(s+1)^{p}
$$


and interchanging the order of the summation and the integration, we have

$$
I_{2}=\frac{\lambda^{n+1}}{(\lambda+1)^{n+2}} \sum_{i=0}^{r}\left(\begin{array}{l}
r \\
i
\end{array}\right)(-1)^{i} \sum_{p=k}^{n} \frac{(\lambda+1)^{p+1}}{i+p+1}
$$

Similarly,

$$
I_{1}=\sum_{i=0}^{n-k} \frac{\lambda^{n-i}}{(\lambda+1)^{n+1-k-i}} \sum_{p=0}^{i+r}\left(\begin{array}{c}
i+r \\
p
\end{array}\right)(-1)^{p} \frac{1}{p+k+1} .
$$

The above manipulation are valid when $\lambda$ is any real number different from -1 . So, we have, for $\lambda \neq-1$,

$$
\begin{aligned}
I_{n, k, r}(\lambda)= & (n+r+1) \sum_{i=0}^{n-k} \frac{\lambda^{k+i}}{(\lambda+1)^{i+1}} \sum_{p=0}^{n+r-k-i}\left(\begin{array}{c}
n+r-k-i \\
p
\end{array}\right)(-1)^{p} \frac{1}{p+k+1} \\
& +(n+r+1) \frac{\lambda^{n+1}}{(\lambda+1)^{n+2}} \sum_{i=0}^{r}\left(\begin{array}{l}
r \\
i
\end{array}\right)(-1)^{i} \sum_{p=k}^{n} \frac{(\lambda+1)^{p+1}}{i+p+1}
\end{aligned}
$$

This proves (4). Now, using (4) with $\lambda$ replaced by $\frac{\theta}{1-\theta}$ for $\theta \neq 1$ (because $\frac{\theta}{1-\theta}$ can takes all values except -1$)$, and multiplying both sides with $(1-\theta)^{n}$, then we have

$$
\begin{aligned}
\sum_{m=k}^{n} \frac{\theta^{m}(1-\theta)^{n-m}}{\left(\begin{array}{c}
n+r \\
m
\end{array}\right)}= & (n+r+1) \sum_{i=0}^{n-k} \theta^{n-i}(1-\theta)^{n-k+1} \sum_{p=0}^{r+i}\left(\begin{array}{c}
r+i \\
p
\end{array}\right)(-1)^{p} \frac{1}{p+k+1} \\
& +(n+r+1) \theta^{n+1} \sum_{i=0}^{r}\left(\begin{array}{l}
r \\
i
\end{array}\right)(-1)^{i} \sum_{p=k}^{n} \frac{(1-\theta)^{n-p}}{i+p+1} .
\end{aligned}
$$

Now, if we compare the coefficients of $\theta^{k}$ on both sides of (7), we can obtain

$$
\frac{1}{\left(\begin{array}{c}
n+r \\
k
\end{array}\right)}=(n+r+1) \sum_{p=0}^{k}\left(\begin{array}{l}
k \\
p
\end{array}\right)(-1)^{k-p} \frac{1}{n+r-p+1} .
$$

In terms of $n+r=s+k$, then we have

$$
\sum_{p=0}^{k}\left(\begin{array}{l}
k \\
p
\end{array}\right)(-1)^{p} \frac{1}{s+p+1}=\frac{1}{(s+k+1)\left(\begin{array}{c}
s+k \\
k
\end{array}\right)} .
$$

To show that (7) is a polynomial identity over $Q$, it is means that both sides of (7) have to coincide in $Q[T]$. Using (9) in (7), we can get the desired result (3). From (6), we can also obtain (5), the proof of which is not detailed here.

Corollary 2.2. For any nonnegative integer $n, k$, and $r$, then

$$
\sum_{m=k}^{n} \frac{(-1)^{n-m}}{\left(\begin{array}{c}
n+r \\
m
\end{array}\right)}=(n+r+1) \sum_{i=0}^{n-k}(-1)^{i} \frac{\left(\begin{array}{c}
n-k+1 \\
i
\end{array}\right)}{\left(\begin{array}{c}
k+r+i \\
k
\end{array}\right)(k+r+i+1)} .
$$


Proof. Comparing the coefficients of $\theta^{n}$ on both sides of (7), we can obtain the desired result.

Theorem 2.3. Let $n$ and $r$ be nonnegative integers. Then

$$
\begin{aligned}
\sum_{m=0}^{n} \frac{1}{\left(\begin{array}{c}
n+r \\
m
\end{array}\right)} & =\sum_{m=0}^{n} \frac{1}{\left(\begin{array}{c}
n+r \\
m+r
\end{array}\right)}=\frac{n+r+1}{2^{n+1}} \sum_{m=0}^{n} \frac{2^{m}}{m+r+1}\left(1+\left(\begin{array}{c}
m+r \\
m
\end{array}\right)^{-1}\right) \\
& =\frac{n+r+1}{2^{n+r}} \sum_{\substack{j \text { odd } \\
\text { i even }}}\left(\begin{array}{c}
n+1 \\
j
\end{array}\right)\left(\begin{array}{l}
r \\
i
\end{array}\right) \frac{1}{i+j} .
\end{aligned}
$$

Proof. The particular case $k=0, \lambda=1$ of (4) in theorem 2.1 gives (11). Now, let us to see the second identity, it follows from identity (1) that

$$
\begin{aligned}
& \sum_{m=0}^{n} \frac{1}{\left(\begin{array}{c}
n+r \\
m
\end{array}\right)}=\sum_{m=0}^{n} \frac{1}{\left(\begin{array}{c}
n+r \\
n-m
\end{array}\right)}=\sum_{m=0}^{n} \frac{1}{\left(\begin{array}{c}
n+r \\
m+r
\end{array}\right)}=(n+r+1) \sum_{m=0}^{n} \int_{0}^{1} t^{m+r}(1-t)^{n-m} d t \\
& =(n+r+1) \int_{0}^{1} t^{r}(1-t)^{n} \sum_{m=0}^{n} t^{m}(1-t)^{-m} d t \\
& =(n+r+1) \int_{0}^{1} t^{r} \frac{t^{n+1}-(1-t)^{n+1}}{2 t-1} d t \\
& =\frac{n+r+1}{2} \int_{-1}^{1}\left(\frac{1+s}{2}\right)^{r} \frac{\left(\frac{1+s}{2}\right)^{n+1}-\left(\frac{1-s}{2}\right)^{n+1}}{s} d s \\
& =\frac{n+r+1}{2^{n+r+2}} \int_{-1}^{1}(1+s)^{r} \frac{(1+s)^{n+1}-(1-s)^{n+1}}{s} d s \\
& =\frac{n+r+1}{2^{n+r+2}} \sum_{j=0}^{n+1}\left(\begin{array}{c}
n+1 \\
j
\end{array}\right)\left(1-(-1)^{j}\right) \int_{-1}^{1}(1+s)^{r} s^{j-1} d s \\
& =\frac{n+r+1}{2^{n+r+2}} \sum_{j=0}^{n+1}\left(\begin{array}{c}
n+1 \\
j
\end{array}\right)\left(1-(-1)^{j}\right) \int_{-1}^{1} \sum_{i=0}^{r}\left(\begin{array}{l}
r \\
i
\end{array}\right) s^{i+j-1} d s \\
& =\frac{n+r+1}{2^{n+r+2}} \sum_{j=0}^{n+1}\left(1-(-1)^{j}\right)\left(\begin{array}{c}
n+1 \\
j
\end{array}\right) \sum_{i=0}^{r}\left(\begin{array}{l}
r \\
i
\end{array}\right) \frac{1+(-1)^{i+j+1}}{i+j} \\
& =\frac{n+r+1}{2^{n+r+1}} \sum_{j \text { odd }}\left(\begin{array}{c}
n+1 \\
j
\end{array}\right) \sum_{i=0}^{r}\left(\begin{array}{l}
r \\
i
\end{array}\right) \frac{1+(-1)^{i}}{i+j} \\
& =\frac{n+r+1}{2^{n+r}} \sum_{j \text { odd }} \sum_{i \text { even }}\left(\begin{array}{c}
n+1 \\
j
\end{array}\right)\left(\begin{array}{l}
r \\
i
\end{array}\right) \frac{1}{i+j} \\
& =\frac{n+r+1}{2^{n+r}} \sum_{\substack{j \text { odd } \\
i \text { even }}}\left(\begin{array}{c}
n+1 \\
j
\end{array}\right)\left(\begin{array}{l}
r \\
i
\end{array}\right) \frac{1}{i+j} .
\end{aligned}
$$

This proves identity (12). 
Then in theorem 2.3, when $r=0$, we obtain (2) directly, and when $r=1, r=2$ or $r=n$, we give the corresponding results as the following corollary.

Corollary 2.4. For any nonnegative integer $n$, then we have

$$
\begin{aligned}
& \sum_{m=0}^{n} \frac{1}{\left(\begin{array}{c}
n+1 \\
m
\end{array}\right)}=\frac{n+2}{2^{n+1}} \sum_{m=0}^{n} \frac{2^{m}}{m+1}=\frac{n+2}{2^{n+1}} \sum_{j \text { odd }} \frac{1}{j}\left(\begin{array}{c}
n+1 \\
j
\end{array}\right), \\
& \sum_{m=0}^{n} \frac{1}{\left(\begin{array}{c}
n+2 \\
m
\end{array}\right)}=\frac{n+3}{2^{n+1}} \sum_{m=0}^{n} 2^{m} \frac{(m+2)(m+1)+2 !}{(m+3)(m+2)(m+1)} \\
& =\frac{n+3}{2^{n+1}} \sum_{j \text { odd }} \frac{j+1}{j(j+2)}\left(\begin{array}{c}
n+1 \\
j
\end{array}\right), \\
& \sum_{m=0}^{n} \frac{1}{\left(\begin{array}{c}
2 n \\
m
\end{array}\right)}=\frac{2 n+1}{2^{n+1}} \sum_{m=0}^{n} \frac{2^{m}}{m+n+1}\left(1+\left(\begin{array}{c}
m+n \\
m
\end{array}\right)^{-1}\right) \\
& =\frac{2 n+1}{2^{2 n}} \sum_{\substack{j \text { odd }, \\
\text { ieven }}}\left(\begin{array}{c}
n+1 \\
j
\end{array}\right)\left(\begin{array}{l}
n \\
i
\end{array}\right) \frac{1}{i+j} .
\end{aligned}
$$

From (11) and (12), we can also obtain the following corollary.

Corollary 2.5. For any nonnegative integer $n$ and $r$, we have

$$
\sum_{m=0}^{n} \frac{2^{m+r-1}}{m+r+1}\left(1+\left(\begin{array}{c}
m+r \\
m
\end{array}\right)^{-1}\right)=\sum_{\substack{j \text { odd }, i \text { even }}}\left(\begin{array}{c}
n+1 \\
j
\end{array}\right)\left(\begin{array}{l}
r \\
i
\end{array}\right) \frac{1}{i+j} .
$$

For example, when $r=0$ or $r=1$, we have

$$
\sum_{m=0}^{n} \frac{2^{m}}{m+1}=\sum_{j \text { odd }} \frac{1}{j}\left(\begin{array}{c}
n+1 \\
j
\end{array}\right) .
$$

When $r=2$, we obtain

$$
\sum_{m=0}^{n} \frac{2^{m}}{m+3}\left(1+\left(\begin{array}{c}
m+2 \\
m
\end{array}\right)^{-1}\right)=\sum_{j \text { odd }} \frac{j+1}{j(j+2)}\left(\begin{array}{c}
n+1 \\
j
\end{array}\right) .
$$

When $r=(k-1) n$, where $k \in N^{+}$, we obtained a rather general theorem.

Theorem 2.6. For any nonnegative integer $n$, and $k \in N^{+}$, then

$$
\sum_{m=0}^{n} \frac{1}{\left(\begin{array}{c}
k n \\
m
\end{array}\right)}=\frac{k n+1}{2^{n+1}} \sum_{m=0}^{n} \frac{2^{m}}{m+(k-1) n+1}\left(1+\left(\begin{array}{c}
m+k n-n \\
m
\end{array}\right)^{-1}\right)
$$




$$
=\frac{k n+1}{2^{k n}} \sum_{\substack{j \text { odd }, \\
\text { ieven }}}\left(\begin{array}{c}
n+1 \\
j
\end{array}\right)\left(\begin{array}{c}
k n-n \\
i
\end{array}\right) \frac{1}{i+j} .
$$

Proof. When $r=(k-1) n$, substituting it into (11) and (12), we can immediately obtain the desired results.

When $k=1$, from (19) we can also obtain the (2). Next, we use another approach to evaluate the sums $\sum_{m=0}^{n} \frac{1}{\left(\begin{array}{c}n+1 \\ m\end{array}\right)}$ and $\sum_{m=0}^{n} \frac{1}{\left(\begin{array}{c}n+2 \\ m\end{array}\right)}$.

Theorem 2.7. For any nonnegative integer $n$, then

$$
\begin{aligned}
\sum_{m=0}^{n} \frac{1}{\left(\begin{array}{c}
n+1 \\
m
\end{array}\right)} & =\frac{n+2}{2^{n+1}} \sum_{j \text { odd }} \frac{1}{j}\left(\begin{array}{c}
n+2 \\
j
\end{array}\right)-1 \\
\sum_{m=0}^{n} \frac{1}{\left(\begin{array}{c}
n+2 \\
m
\end{array}\right)} & =\frac{n+3}{2^{n+2}} \sum_{m=0}^{n} \frac{2^{m}}{m+1}+\frac{1}{2}-\frac{1}{2(n+2)} \\
& =\frac{n+3}{2^{n+2}} \sum_{j \text { odd }} \frac{1}{j}\left(\begin{array}{c}
n+3 \\
j
\end{array}\right)-\frac{1}{n+2}-1 .
\end{aligned}
$$

Proof. First, we have

$$
\sum_{m=0}^{n} \frac{1}{\left(\begin{array}{c}
n+1 \\
m
\end{array}\right)}=\sum_{m=0}^{n+1} \frac{1}{\left(\begin{array}{c}
n+1 \\
m
\end{array}\right)}-\frac{1}{\left(\begin{array}{c}
n+1 \\
n+1
\end{array}\right)}=\frac{n+2}{2^{n+1}} \sum_{j \text { odd }} \frac{1}{j}\left(\begin{array}{c}
n+2 \\
j
\end{array}\right)-1
$$

and

$$
\begin{aligned}
\sum_{m=0}^{n} \frac{1}{\left(\begin{array}{c}
n+2 \\
m
\end{array}\right)} & =\sum_{m=0}^{n+2} \frac{1}{\left(\begin{array}{c}
n+2 \\
m
\end{array}\right)}-\frac{1}{\left(\begin{array}{c}
n+2 \\
n+1
\end{array}\right)}-\frac{1}{\left(\begin{array}{c}
n+2 \\
n+2
\end{array}\right)}=\frac{n+3}{2^{n+2}} \sum_{m=0}^{n+2} \frac{2^{m}}{m+1}-\frac{1}{n+2}-1 \\
& =\frac{n+3}{2^{n+2}}\left(\frac{2^{n+2}}{n+3}+\frac{2^{n+1}}{n+2}+\sum_{m=0}^{n} \frac{2^{m}}{m+1}\right)-\frac{1}{n+2}-1 \\
& =\frac{n+3}{2^{n+2}} \sum_{m=0}^{n} \frac{2^{m}}{m+1}+\frac{1}{2}-\frac{1}{2(n+2)} .
\end{aligned}
$$

On the other hand,

$$
\sum_{m=0}^{n} \frac{1}{\left(\begin{array}{c}
n+2 \\
m
\end{array}\right)}=\sum_{m=0}^{n+2} \frac{1}{\left(\begin{array}{c}
n+2 \\
m
\end{array}\right)}-\frac{1}{\left(\begin{array}{c}
n+2 \\
n+1
\end{array}\right)}-\frac{1}{\left(\begin{array}{c}
n+2 \\
n+2
\end{array}\right)}=\frac{n+3}{2^{n+2}} \sum_{j \text { odd }} \frac{1}{j}\left(\begin{array}{c}
n+3 \\
j
\end{array}\right)-\frac{1}{n+2}-1,
$$

which conclude the result.

From (13), (14), (20), (21) and (22), after some computation, we can obtain the following result.

Theorem 2.8. For any nonnegative integer $n$, then

$$
\sum_{m=0}^{n} 2^{m} \frac{m^{2}+m+2}{(m+3)(m+2)(m+1)}=\frac{(n+1) 2^{n+1}}{(n+2)(n+3)} .
$$




$$
\begin{gathered}
\sum_{\substack{j \text { odd }, j \leq n+2}} \frac{1}{j}\left(\begin{array}{c}
n+1 \\
j-1
\end{array}\right)=\frac{2^{n+1}}{n+2} . \\
\sum_{\substack{j \leq \text { odd }, j \leq n+3}} \frac{1}{j}\left(\left(\begin{array}{c}
n+3 \\
j
\end{array}\right)-\frac{2(j+1)}{j+2}\left(\begin{array}{c}
n+1 \\
j
\end{array}\right)\right)=\frac{2^{n+2}}{n+2} .
\end{gathered}
$$

Proof. From the first equation of (14) and (21), we can get (23). From the second equation of (13) and (20), we obtain (24) easily. From the second equation of (14) and (22), we obtain (25).

\section{Acknowledgements}

The authors would like to thank the anonymous referee for his/her valuable suggestions. This research is supported by the National Natural Science Foundation of China (Grant No. 10771093), the Natural Science Foundation of Henan Province (Grant No. 0511010300).

\section{References}

[1] H. W. Gould, Combinatorial identities, West Virginia University, 1972.

[2] A. Sofo, General properties involving reciprocals of bonomial coefficients, Journal of Integer Sequence 9(2006), Article 06.4.5.

[3] B. Sury, Sum of the reciprocals of the binomial coefficients, European J. Combin. 14.4 (1993), 351353.

[4] T. Trif, Combinatorial sums and series involving inverses of binomial coefficients, Fibonacci Quart. 38.1(2000), 79-84.

[5] J. H. Yang and F. Z. Zhao, Sums involving the inverses of binomial coefficients, Journal of Integer Sequence, 9(2006), Article 06.4.2.

[6] F. Z. Zhao and T. M. Wang, Some results for sums of the inverses of binomial coefficients, Integers: Electronic Journal of Combinatorial Number Theory, 5(2005), A22.

[7] B. Sury, T. Wang and F. Z. Zhao, Identities involving reciprocals of bonomial coefficients, Journal of Integer Sequence, 7(2004), Article 04.2.8.

Department of Mathematics, Luoyang Teachers' College, Luoyang 471022, P. R. China.

College of Mathematics and Information Science, Henan University, Kaifeng 475001, P. R. China.

E-mail: zhzhzhang-yang@163.com 